\title{
Comparative analysis of 4C-Seq data generated from enzyme-based and sonication-based methods
}

Fan Gao ${ }^{1,2}$, Zong Wei ${ }^{1}$, Wange $\mathrm{Lu}^{1 *}$ and Kai Wang ${ }^{2^{*}}$

\begin{abstract}
Background: Circular chromosome conformation capture, when coupled with next-generation sequencing (4C-Seq), can be used to identify genome-wide interaction of a given locus (a "bait" sequence) with all of its interacting partners. Conventional $4 \mathrm{C}$ approaches used restriction enzyme digestion to fragment chromatin, and recently sonication approach was also applied for this purpose. However, bioinformatics pipelines for analyzing sonication-based 4C-Seq data are not well developed. In addition, data consistency as well as similarity between the two methods has not been explored previously. Here we present a comparative analysis of 4C-Seq data generated by both methods, using an enhancer element of Pou5f1 gene in mouse embryonic stem (ES) cells.
\end{abstract}

Results: From biological replicates, we found good correlation ( $r>0.6)$ for inter-chromosomal interactions identified in either enzyme or sonication method. Compared to enzyme approach, sonication method generated less distal intra-chromosomal interactions, possibly due to the difference in chromatin fragmentation. From all mapped interactions, we further applied statistical models to identify enriched interacting regions. Interestingly, data generated from the two methods showed 30\% overlap of the reproducible interacting regions. The interacting sites in the reproducible regions from both methods are similarly enriched with active histone marks. In addition, the interacting sites identified from sonication-based data are enriched with ChIP-Seq signals of transcription factors Oct4, Klf4, Esrrb, Tcfcp2i1, and Zfx that are critical for reprogramming and pluripotency.

Conclusions: Both enzyme-based and sonication-based 4C-Seq methods are valuable tools to explore long-range chromosomal interactions. Due to the nature of sonication-based method, correlation analysis of the $4 \mathrm{C}$ interactions with transcription factor binding should be more straightforward.

Keywords: Circular chromosome conformation capture, Sonication, 4C-Seq, Bioinformatics, Next-generation sequencing

\section{Background}

Chromosomal organization in the nucleus has been gradually recognized as an important contributor to gene regulation and genome function. In the last decade, chromosome conformation capture (3C) has been developed as a high-throughput assay to explore longrange chromatin-chromatin interactions in vivo [1]. Technologies derived from $3 \mathrm{C}$, such as $\mathrm{Hi}-\mathrm{C}[2,3], 5 \mathrm{C}$

\footnotetext{
* Correspondence: wangelu@usc.edu; kaiwang@usc.edu

${ }^{1}$ Eli and Edythe Broad Center for Regenerative Medicine and Stem Cell Research, Department of Biochemistry and Molecular Biology, University of Southern California, Los Angeles, CA 90089, USA

${ }^{2}$ Zilkha Neurogenetic Institute, Department of Psychiatry and Department of Preventive Medicine, University of Southern California, Los Angeles, CA 90089, USA
}

\section{Biomed Central}

(c) 2013 Gao et al.; licensee BioMed Central Ltd. This is an Open Access article distributed under the terms of the Creative Commons Attribution License (http://creativecommons.org/licenses/by/2.0), which permits unrestricted use, distribution, and reproduction in any medium, provided the original work is properly cited.
[4] and TCC [5], explore global chromatin interactions. Other methods like ChIP-3C [6-10] and ChIA-PET (chromatin interaction analysis by paired-end tag sequencing) detect chromatin-chromatin interactions mediated by a specific DNA binding protein [11-13]. A particular derivative of $3 \mathrm{C}$ method named circular chromosome conformation capture (4C) enables de novo detection of all interacting partners of a known genomic region, such as differentially methylated $\mathrm{H} 19$ imprinting control region [14]. Similar to 3C-based global mapping of chromatinchromatin interactions, $4 \mathrm{C}$ follows the same concept of 1 ) crosslinking of protein-DNA complexes in the cell nucleus to capture chromosome conformation and 2) proximity ligation of physically interacting chromatin fragments. 
However, instead of using immuno-precipitation to capture the ligated junction DNA pieces in ChIP-3C and ChIAPET, 4C utilizes PCR reactions to enrich genomic regions interacting with a known "bait" region. The PCR products generated from the $4 \mathrm{C}$ technology can be subsequently examined by different approaches: they can be cloned for Sanger sequencing [15], or hybridized to a microarray $[14,16]$. Given the rapid development of next-generation sequencing, it is now feasible to directly interrogate the PCR products from the $4 \mathrm{C}$ technology (hereafter referred to as "4C-Seq"). This technique enables genome-wide mapping of the chromatins interacting with the "bait" in greater resolution and precision.

In most of the published $4 \mathrm{C}$ studies to date [14,16-20], 4C library preparation typically starts with formaldehyde crosslinking of chromosomes in vivo, followed by restriction enzyme digestion to fragment chromosomes. After proximity ligation in diluted condition, chromatin proteins are digested with proteinase $\mathrm{K}$ and formaldehyde crosslinks are reversed. Finally "bait"-containing circular DNA molecules generated from the ligation are amplified using bait-specific primers in nested PCR reactions. Although enzyme digestion-based $4 \mathrm{C}$ method has limitations such as noisy/weak chromatin associations [21,22], low chromatin accessibility-dependent digestion efficiency as well as uneven distribution of restriction digestion sites across the genome, it should be acknowledged that recent advance in enzyme-based 4C method greatly increased data resolution and robustness by using 4-bp cutter instead of 6-bp cutter in fragmentation [23]. Compared to enzyme digestion, sonication is less accessibility-dependent and preferentially breaks crosslinked chromosomes at the edge of protein binding sites [24,25]. Thus sonication-based approach provides an alternative choice for 4C-Seq studies [26], which is potentially more straightforward on exploring bound transcription factor(s) that mediate the interactome.

Unlike enzyme digestion-based 4C method [17-19], sonication-based library presents a data analysis challenge which requires a different set of analytical approaches. The major reason is that each restriction enzyme has a set of known breakage sites in the genome, so that researchers typically align all reads against flanking sequences of this set of restriction enzyme site, and the restriction sites are the exact ligation sites between two interacting regions [18]. In contrast, sonication method has generally no preference of sequence motifs at breakage sites. Without knowing the exact ligation sites a priori, the pipeline for data processing is anticipated to be different from enzyme digestion-based method. So far, the bioinformatics pipelines have not been well developed to process sonication-based $4 \mathrm{C}$ data generated by high-throughput short-read sequencers, such as Illumina Hi-Seq. In the current study, we describe a sonication-based 4C-Seq protocol that we applied to explore Pou5f1 enhancer interactome in mouse ES cells. The
Pou5f1 enhancer has been known to mediate expression of its own gene product - Oct4, a key reprogramming factor [27]. Thus exploration of this enhancer interactome will provide better understanding of Pou $5 f 1$-related regulatory network. To compare different $4 \mathrm{C}$ protocols, we also performed a parallel study using an enzyme-based method described in [18]. We analyzed consistency of processed 4C-Seq data generated from biological replicates, applied statistical analysis to identify enriched interacting regions, compared the reproducible enriched interacting regions identified from enzyme and sonication methods, and explored epigenetic features enriched in the $4 \mathrm{C}$ interactome.

\section{Results and discussion}

\section{Overview of sonication-based $4 \mathrm{C}$ library preparation}

Based on previously published $4 \mathrm{C}$ studies on KRT gene cluster [26], we slightly modified the experimental protocol for sonication-based 4C library preparation (Figure 1, see Methods for details). We chose an upstream enhancer element of Pou5f1 gene in mouse ES cells as bait and constructed $4 \mathrm{C}$ libraries for two biological replicate samples BR1 and BR2. Compared to the study by Huang et al. [26], our method on post-processing of the generated $4 \mathrm{C}$ library for next-generation sequencing is notably different. Huang et al. analyzed the amplified 4C products consisting of bait-target-bait DNA pieces using 454 Titanium Sequencer. In our study, to adapt to Illumina short-read sequencing, an additional sonication step fragments $4 \mathrm{C}$ products into 200 bp DNA pieces containing bait, targets, baittarget junctions as well as genomic contaminants, before subject to Illumina $\mathrm{Hi}$-Seq sequencing. Sonication of ligated DNA fragments to smaller pieces for Hi-Seq sequencing was also applied in a recent $\mathrm{Hi}-\mathrm{C}$ study [28]. We used an end-tag mapping method, similar to the one described in [24] for identifying junction reads from sonication-based 4C-Seq data. In our study, enzyme-based 4C-Seq library preparation strictly followed the protocol described in [18].

\section{Data processing framework}

Based on our experimental protocol, the short sequencing reads from Illumina $\mathrm{Hi}$-Seq platform should theoretically fall into four categories: 1) reads at the $4 \mathrm{C}$ bait locus, 2) reads at the bait interacting regions, 3) reads that spans ligation junctions between bait locus and its interacting region, as well as 4) noises caused by contamination of genomic or circular DNA that are not amplified in PCR reactions (Figure 1A). We therefore evaluated different strategies to identify these four types of reads.

We first attempted to separately map both forward and reverse reads (91 bp) of the paired-end data to the reference genome (mm9) using Burrows-Wheeler Aligner (BWA [29]), to identify the reads that are completely aligned to the "bait" locus or other genomic regions. For sequencing data generated from the two biological replicates, the mapped 
reads account for $\sim 77 \%$ of the total reads (Additional file 1: Table S1), with most of the mapped reads being uniquely mapped. With this mapping strategy, >99\% of the uniquely mapped reads are within the bait locus, suggesting the presence of many proximal interactions or self-ligations in the data. Only $0.2 \%$ to $0.6 \%$ of the uniquely mapped reads are mapped to distal genomic regions, that is, the majority of them should fall into Category 2 described above, though it is possible that some of them may originate from genomic DNA described in Category 4. We also note that unmapped reads in the biological replicates account for $\sim 23 \%$ of total reads, which may correspond to ligation junctions that cannot be mapped to the reference genome, that is, Category 3 above. Given the limited amount of data supporting Category 2, we believe that this mapping strategy is not optimal for identifying bait interacting regions.

We next evaluated an end-tag mapping strategy $[2,11]$, generally applied in 3C-based studies to identify Category 3 reads that are mosaic of the bait and its interacting regions. A similar strategy was also previously used in a ChIA-PET study [30], with 20-bp end tags. Here we define "bait region" as a $\sim 1 \mathrm{~kb}$ region, which includes $500 \mathrm{bp}$ extension from the locations of the 2nd set of forward and reverse PCR primers (Figure 1). We extracted 20-bp end tags from both forward and reverse sequencing reads and aligned them to the reference genome assembly separately using BWA [29]. The generated forward and reverse alignment files were merged together using SAMtools [31]. Junction reads are identified, when one end-tag uniquely maps to the "bait" and the other end-tag maps to genomic locations $>300 \mathrm{bp}$ away on the same chromosome (intra-chromosomal interactions) or to a different chromosome (inter-chromosomal interactions). The rationale to choose $300 \mathrm{bp}$ is that our sonication approach generates small DNA pieces with an average size of $200 \mathrm{bp}$ for sequencing, so end-tags that are > 300 bp away should mostly be junction reads. We next classified junction reads as proximal junction reads and distal junction reads. Proximal junction reads have two end tags mapped on the same chromosome with genomic distance between the tags between $300 \mathrm{bp}$ and $10 \mathrm{~kb}$. Distal junction reads are either two tags on the same chromosome with distance greater than $10 \mathrm{~kb}$ or two tags on different chromosomes. Proximal junction reads account for $~ 90 \%$ of total junction reads identified (Additional file 1: Table S2), with the distribution of relative genomic distance between the two ends following a continuous decay, starting from $300 \mathrm{bp}$ to $2 \mathrm{~kb}$, similar to the self-ligation events observed in a ChIA-PET study [30]. Predominant proximal ligation may reflect disruption of weak chromatin-chromatin interactions under the shearing force, thus facilitating self-ligation events. Since no interactions were identified within the distance range from $2 \mathrm{~kb}$ to $10 \mathrm{~kb}$ in both BR1 and BR2 biological replicate data, we used $10 \mathrm{~kb}$ distance cutoff to distinguish proximal $v s$. distal intra-chromosomal interactions.
To explore distal chromatin-chromatin interactions, we processed distal junction reads for further analysis. Tags that were within $100 \mathrm{bp}$ range on their genomic locations were considered as PCR products from a single ligation event and merged as one unique distal interacting site, given that the $4 \mathrm{C}$ library DNA was fragmented before sequencing. Unique distal interacting sites supported by only one read were removed from our analysis as they likely represent background noises. In total, using $20 \mathrm{bp}$ end-tag mapping, we identified 5,705 and 4,368 filtered unique interacting sites respectively from two biological replicate data generated from sonication-based $4 \mathrm{C}$ method. In the following sections, we will discuss $4 \mathrm{C}$ sequencing data in the context of data reproducibility, effect of sequencing depth, statistical models for identifying enriched interacting regions, comparison of reproducible interacting regions identified in both enzyme and sonication methods, epigenetic histone features surrounding the interacting sites within the reproducible regions, and transcription factors enriched around sonication generated interacting sites.

\section{Reproducibility of the inter-chromosomal interactions}

Chromatin-chromatin interactions are highly dynamic [32], and the interactions are probably more transient than protein-chromatin interactions in the nucleus. For example, CTCF mediated interactome in mouse ES cells has only $38 \%$ overlap between biological replicates, suggesting dynamic feature of CTCF mediated chromatin-chromatin interactions; while $98 \%$ peaks identified in CTCF ChIA-PET study can be found in ChIP-Seq peak data of CTCF, reflecting strong association of CTCF with chromatin fibers [12]. We note that the $4 C$-Seq approach aims to take a snapshot of chromatin interacting patterns, which reflect the average state across hundreds of thousands of cells. Genome-wide 3C/Hi-C, ChIA-PET studies revealed more consistent proximal interactions than distal interactions. It is likely that sequencing depth for such studies is not sufficient for capturing less frequent distal interactions, such as interchromosomal interactions. Compared to 3C-based studies, $4 \mathrm{C}$-Seq explores the interactions associated with only one bait area, thus in theory, it enables a more thorough search of less frequent long-range interactions, provided the same sequencing depth of the prepared DNA libraries. Therefore, we decided to determine the reproducibility of interchromosomal interactions between biological replicate samples by counting the number of observed interactions in every genomic bin and calculate the correlation between replicates. The correlation of the data is an indication whether the bait region has preferred interacting partners located on different chromosomes or the interchromosomal interactions mainly result from random collision between the chromosomes. As for 4C-Seq data, the reproducibility can also be affected by many factors including efficiency of proximity ligation, PCR 
amplification, DNA fragmentation, the quality and depth of next-generation sequencing, as well as the data processing strategy. Theoretically, sticky-end ligation applied in enzyme-based method should result in higher ligation efficiency than blunt-end ligation in sonicationbased method; however, smaller size of chromatin fragments generated by sonication may contribute to higher chance of collision frequency between two breakage points. In our analysis, the replicate interactome data of the Pou $5 f 1$ enhancer generated from both methods were included for comparison (Additional file 2: Figure S1). The recommended data processing protocol for analyzing enzyme-based inter-chromosomal interactions [18] was used to count the number of ligated HindIII sites for each genomic segment that covers 500 HindIII sites. For 4C-Seq data generated by sonication method, we counted the number of identified unique interacting sites in each $2 \mathrm{Mb}$ genomic bin (roughly the size covering 500 of 6-bp cutters) to explore the correlation between biological replicates. For inter-chromosomal interactions generated in biological replicate mouse ES cells, we found Pearson's correlation coefficient values of 0.658 and 0.636 for enzyme-based and sonication-based $4 \mathrm{C}$ libraries respectively (Figure 2). When we attempted to use smaller bin sizes (1 Mb and $500 \mathrm{~kb}$ ) for

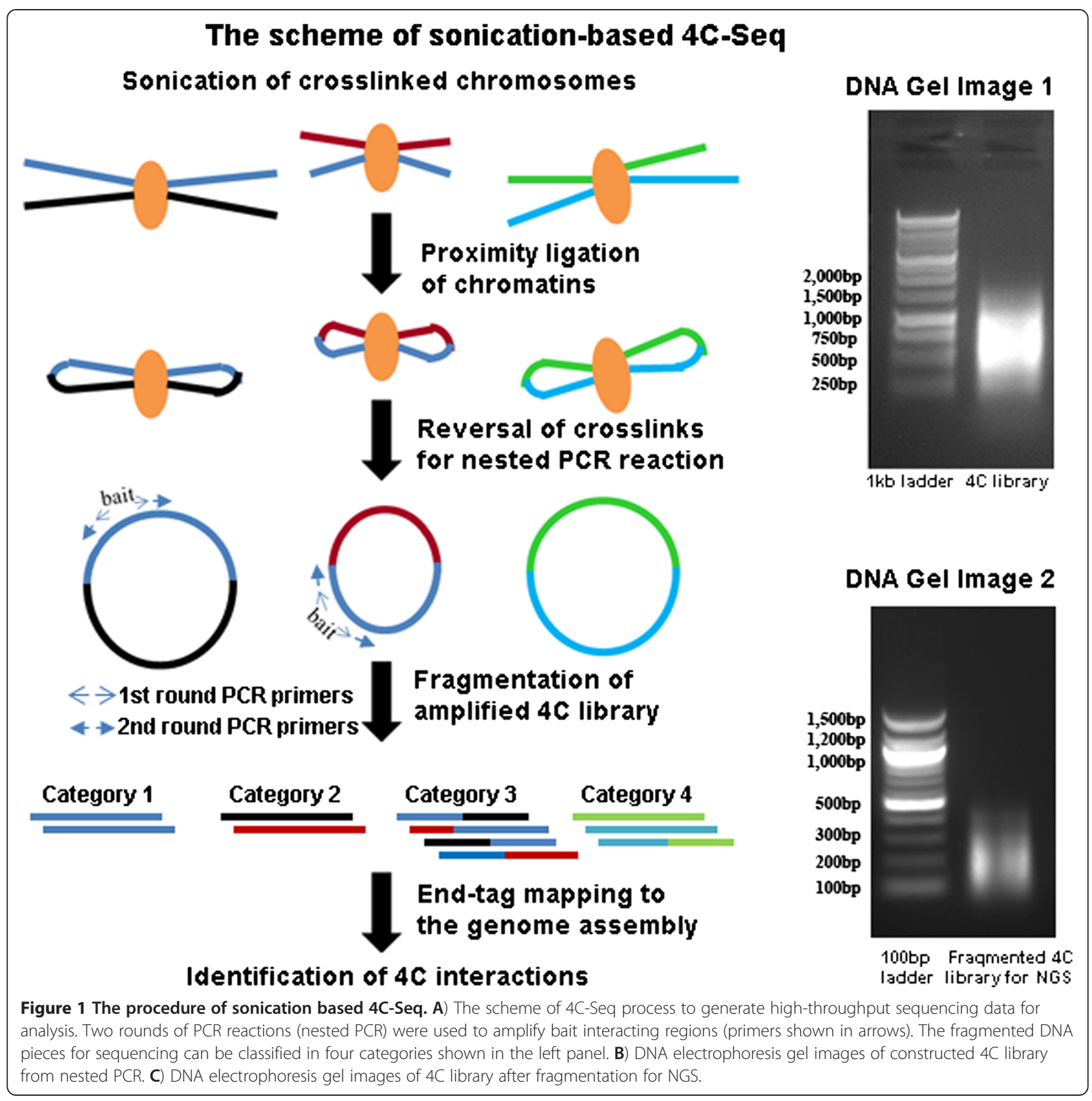



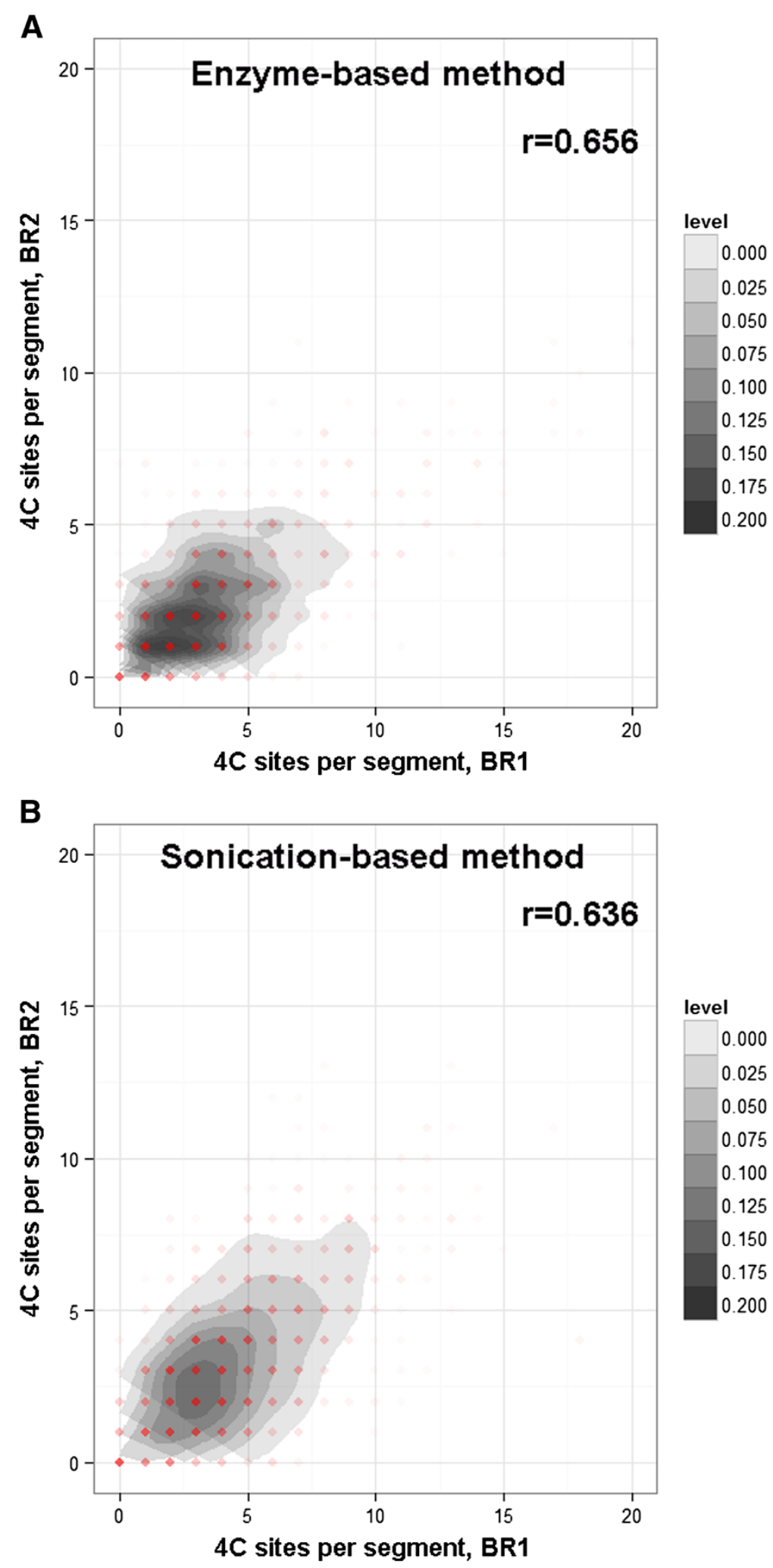

Figure 2 Reproducibility of inter-chromosomal interactions. Density scatter plots of inter-chromosomal interactions identified in the biological replicate data for both sonication-based and enzyme-based methods. Pearson's correlation coefficient values were also shown in the upper right corner of the plots. 
sonication-based data, the correlation coefficient between the replicates decreased to 0.559 and 0.473 respectively (Additional file 3: Figure S2A\&C). For enzyme-based data, using smaller bin sizes (250 and 125 6-bp sites) also resulted in a similar decrease of correlation coefficient values $(\mathrm{r}=0.581, \mathrm{r}=0.475$; Additional file 3: Figure S2B\& D). Thus, the two methods showed similar reproducibility, and sonication-based method doesn't improve or degrade the resolution in this case. Also in our experiments, the biological replicate data for enzyme-based 4C libraries showed that $36 \%$ of the inter-chromosomal interactions identified in the second replicate are in close proximity (within $10 \mathrm{~kb}$ range) to the interactions in the first replicate, whereas $46 \%$ of the inter-chromosomal interactions from sonicationbased 4C libraries showed proximity between biological replicate. This analysis indicated that interacting regions identified from biological replicates are relatively consistent with each other, in spite of different methods applied in chromatin fragmentation.

\section{Evaluation of sequencing depth}

Intuitively, sequencing depth is directly related to the ability to find relatively rare interacting events, as well as the true fraction of the reads that can be informative to finding distal interacting events. We explored with $10 \%, 25 \%, 50 \%$, $75 \%, 90 \%$ and $100 \%$ of the original sequencing data to perform the same set of analysis for the replicate data of sonication-based 4C libraries, and analyzed their correlation patterns. As shown in Figure 3, with sequencing depth rose from $10 \%$ to $100 \%$, the coverage of identified clustered distal interacting sites in the two biological replicates (BR1 and BR2) gradually increased from $30 \%$ to $46 \%$ (coverage defined as the percentage of BR2 sites within $10 \mathrm{~kb}$ of BR1 sites). More importantly, pairwise correlation of interacting frequencies of $2 \mathrm{Mb}$ genomic bins between BR1 and BR2 showed an increment from 0.328 to 0.636 . However, when more than $75 \%$ of the sequencing reads were used in analysis, both coverage of the interacting sites and correlation of the interacting frequencies in the two replicates reached a plateau. Therefore, $\sim 20$ million total short reads (10 million read pairs) from Illumina sequencing is sufficient to capture most of the interacting events of this enhancer element in mouse ES cells.

Statistical analysis to identify enriched interacting regions from the inter-chromosomal interactions

As a high-throughput assay, 4C-Seq revealed thousands of sites interacting with one bait region. However, it is unlikely that all the interactions identified are biologically significant, and many of them probably represent random collision between two genomic fragments in 3D space. To identify regions that are frequently associated with the bait region other than random collision, we applied statistical models to analyze interacting sites within each chromosome. We used a permutation-based false discovery rate (FDR) procedure to choose significantly enriched interacting regions.

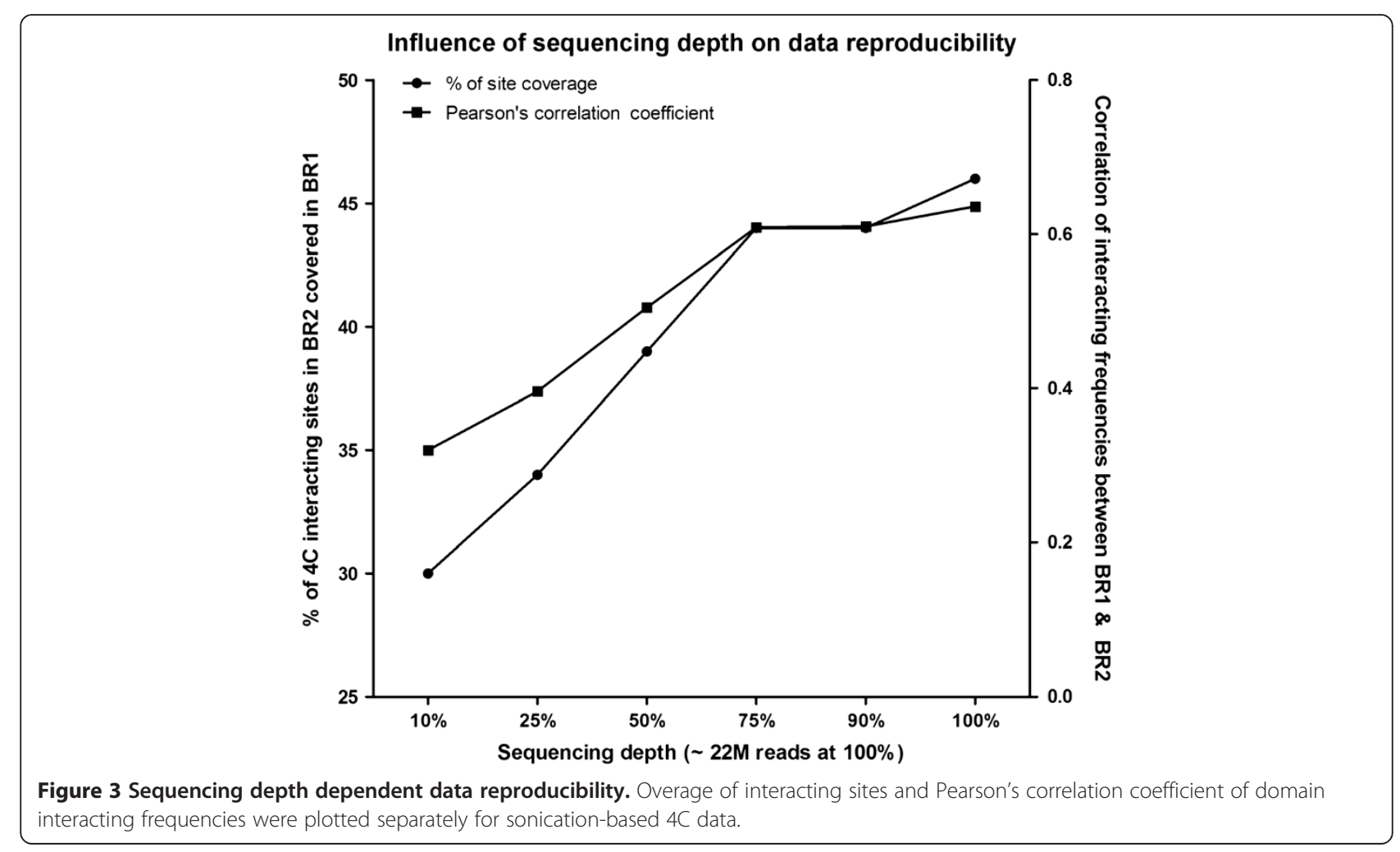


For enzyme-based 4C data, a z-score was assigned based on the number of $4 \mathrm{C}$ interacting sites per 500 HindIII sites [18]. For sonication-based data, each interacting site was assigned a z-score based on the nearby interactions observed within $\pm 1 \mathrm{Mb}$ distance range (see Methods for details). FDR was calculated by random permutation of the data 100 times, and the cutoff value of $5 \%$ was used to select positive sites. Positive sites and the nearby interaction sites $( \pm 1 \mathrm{Mb}$ range) were grouped together as enriched interacting regions. Overlapping enriched regions were further merged together. The statistical models applied here aim to identify enriched interacting regions from the background, similar to the concept used in ChIP-Seq peak calling.

\section{Comparison of enriched interacting regions identified from 4C-Seq data generated from different methods}

Statistical analysis identified 65 and 82 enriched interchromosomal interacting regions for each replicate data generated from the enzyme approach, with about $40 \%$ overlap of the identified regions (30 regions) between the two biological replicates (Figure 4A). For sonication-based data, 76 and 85 regions were identified from each replicate, with nearly $50 \%$ of the regions are overlapping between the two replicates (Figure 4B). Thus those reproducible regions represent high-confidence interactions that might have biological consequences. It is interesting to note that one third of the reproducible inter-chromosomal regions identified in the enzyme method overlap with the reproducible regions from sonication-based data (Figure 4C). Thus, the Pou5f1 enhancer shows preference in interacting with distal regions that are located on different chromosomes, and the identified reproducible regions even possess certain level of consistency between sonication-based and enzyme-based 4C-Seq approaches.

\section{Comparison of distal intra-chromosomal interactions}

Intra-chromosomal interactions include both proximal cis-interactions around the bait locus and long-range cisinteractions distal to the bait area. Both enzyme and sonication-based 4C-Seq data revealed a majority of proximal cis-interactions in all the interaction reads identified (Additional file 1: Table S2 \& S3). As shown in the distribution plot for the identified intra-chromosomal interaction reads (Figure 5), distal interactions occur even at $60 \mathrm{Mb}$ away from the bait location. Interesting to note, sonication-based method generated less distal intrachromosomal interactions compared to enzyme-based 4C-Seq method (Figure 5). For enzyme-based 4C-Seq, $18.5 \%$ and $12.6 \%$ of all the HindIII sites on Chromosome 17 were identified as interaction sites for the two biological replicate data respectively, suggesting a high background (Figure 5B) in intra-chromosomal interactions, similar to the observations published by de Latt group [18]. Data

\section{Enzyme-based}

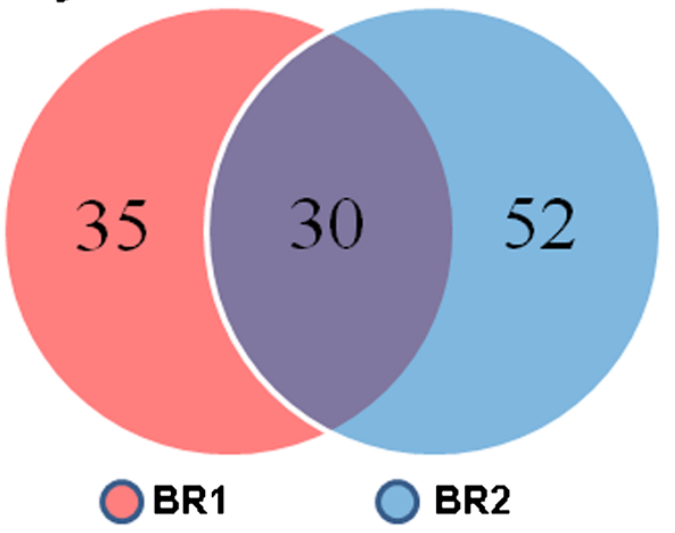

\section{Sonication-based}

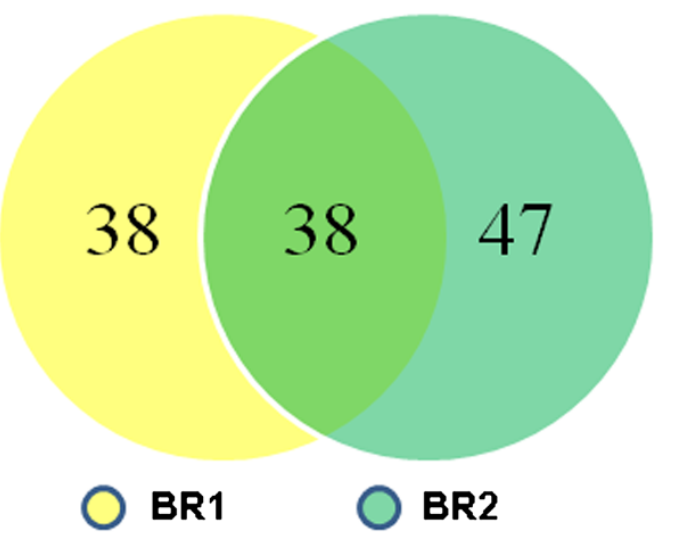

Reproducible regions

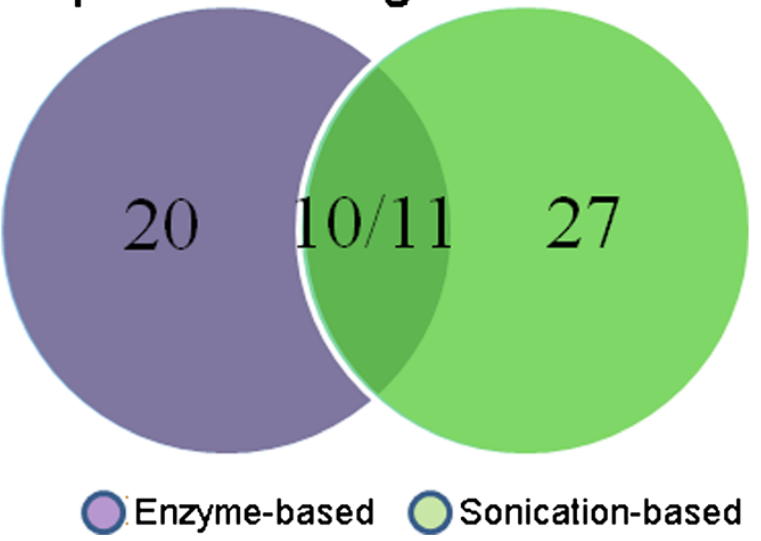

Figure 4 Overlap of inter-chromosomally enriched interacting regions. $\mathbf{A}, \mathbf{B}$ ) Overlap of enriched inter-chromosomal interacting regions identified in the biological replicate data generated using enzyme-based or sonication-based 4C-Seq approaches; C) Overlap of reproducible inter-chromosomal interacting regions between the two different 4C-Seq methods. 


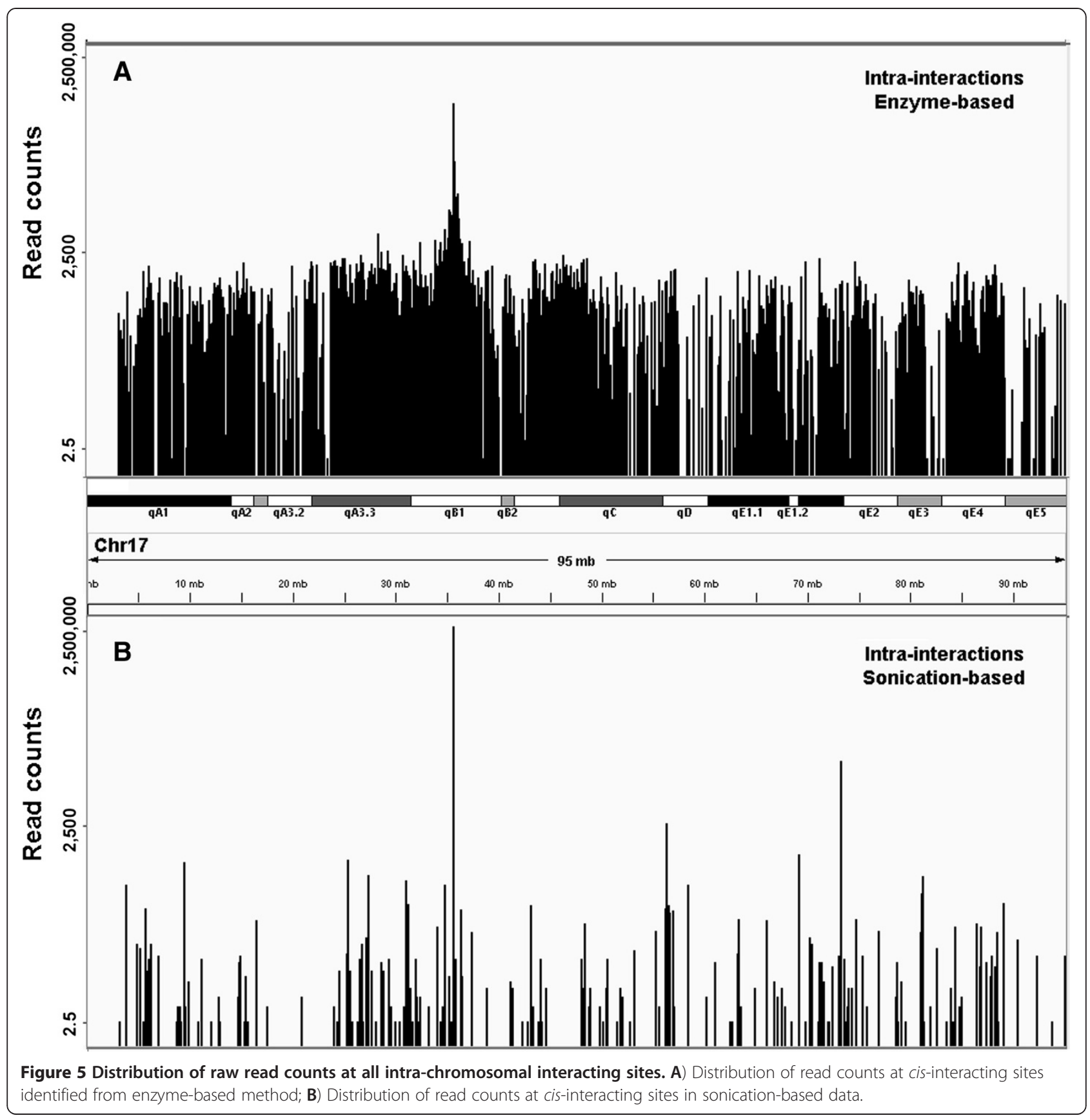

generated by sonication-based method is consistent with the 4C-Seq data from Ruan group with only a few distal intra-chromosomal interactions [11]. Clearly, in our case, we did not observe predominant distal intrachromosomal interactions within all the distal interactions from sonication-based method, contrasting to the data generated from the enzyme method we used. Sequencing depth in general, affects interactions identified; however, since we are examining ratio of distal intra-chromosomal interactions among all interactions, differences in sequence coverage is unlikely to play a major role. Interesting to note, a previous e4C study [33] showed predominant inter-chromosomal interactions of $\mathrm{Hbb}$ locus by using an array-based $4 \mathrm{C}$ technique. We suspect that sonicationbased method may have shaken off a lot of weak interactions [22] that were identified in the enzyme-based approach we applied.

For enzyme-based 4C-Seq data, we followed a statistical model [18] to identify enriched cis-interacting regions. In brief, $\mathrm{z}$-scores in windows covering 100 enzyme sites (size $\sim 400 \mathrm{~kb}$ ) were calculated based on contact frequencies in each window, with a background 
window covering 3,000 sites to calculate expected contact frequency. A permutation-based FDR method with a threshold (FDR $\leq 5 \%)$ was used to select enrichedinteracting regions as described above for the interchromosomal interactions. Shown in Figure 6, the coverage between the biological replicate data is above $80 \%$. For sonication-based method, windows of $\pm 200 \mathrm{~kb}$ around identified contact sites were used to identify enrichedinteracting regions (see methods). The overlap of enrichedinteracting regions between the two replicate data is $\sim 33 \%$, lower than enzyme-based method. Still, $30 \%$ of the reproducible regions overlapped between the two methods.

\section{Epigenetic histone marks enriched in the interactomes}

Previous $\mathrm{Hi}-\mathrm{C}$ study unveiled chromosomal organization of open and closed chromatin compartments in the cell nucleus [2], with open chromatin compartments enriched with active epigenetic features. We questioned whether the interactomes of active Pou $5 f 1$ enhancer are associated with specific epigenetic features. Thus we performed association study to calculate enrichment factors for a series of histone marks in the interactomes. For both enzymebased and sonication-based data, histone marks related to gene activation, such as H3K27ac, H3K36me3, H3K4me1, H3K4me3 and H3K9ac were enriched around the identified contact sites $( \pm 5 \mathrm{~kb}$ range) within the reproducible interacting regions (Figure 7). In contrast, enrichment for H3K27me3 repressor mark and H3K9me3 heterochromatin mark was either not obvious or not observed at all. Thus in our study, 4C-Seq data revealed physical proximity of an enhancer element with distally active genomic regions in mouse ES cells, consistent with the concept of active genomic compartments from genome-wide $3 \mathrm{C}$ studies [2,5]. These results therefore serve as positive controls for our experiments, and confirmed that biological insights can be inferred from both enzyme-based and sonication-based 4C-Seq data sets with appropriate analytical approaches.

\section{Transcription factors enriched in the interactomes}

Unlike enzyme-based method, sonication-based 4C method applied ultrasound to shear cross-linked chromosomes into smaller pieces, similar to the fragmentation step in ChIPSeq protocol. If particular DNA-binding protein complexes are involved in mediating chromosomal interactions between the bait and other distal regions, the identified baitinteracting sites from sonication-based data should be in close proximity to the bound sites of those DNA-binding proteins. We analyzed ChIP-Seq raw read files of 15 DNAbinding proteins reported in Chen et al's study [34]. Briefly, the read tags within $\pm 1 \mathrm{~kb}$ range of a $4 \mathrm{C}$ site was counted and normalized as having 10 million total read tags. The input data for the study was used to generate normalized background read counts. Specific ChIP-Seq read counts

\section{Enzyme-based}

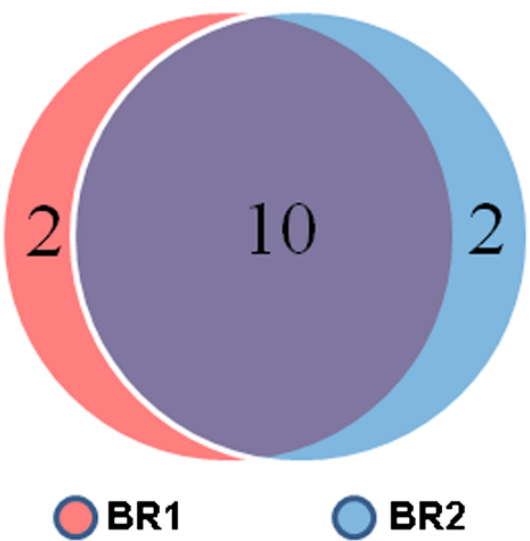

\section{Sonication-based}

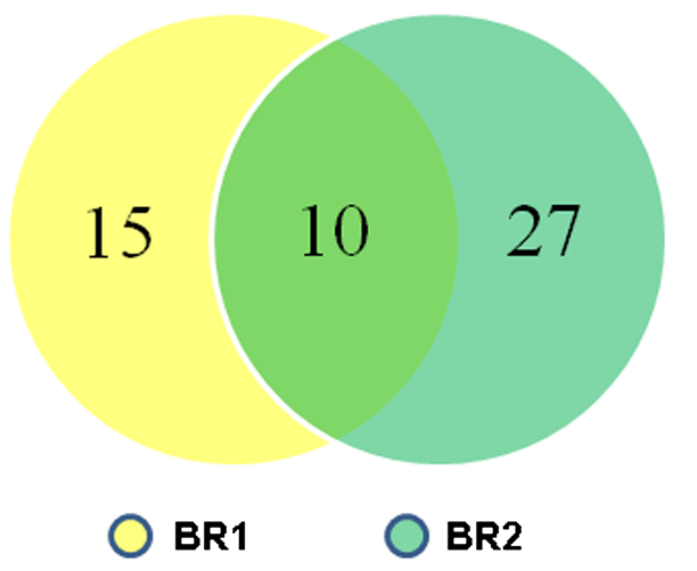

\section{Reproducible regions}

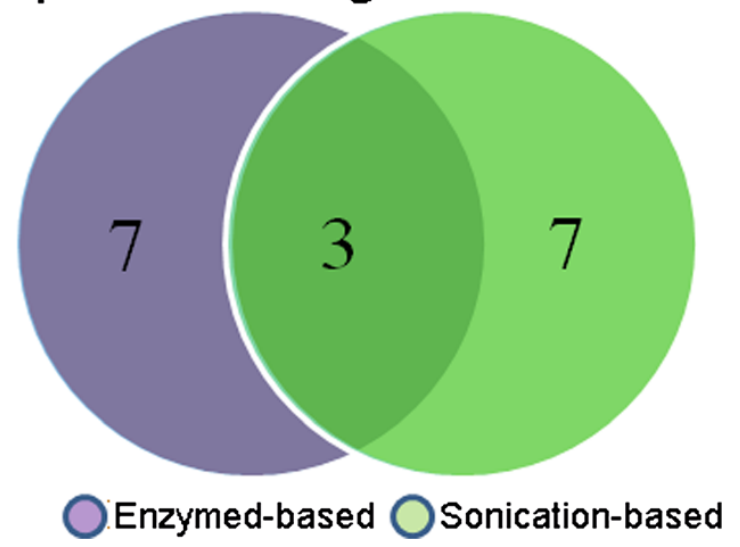

Figure 6 Overlap of intra-chromosomally enriched interacting regions. A,B) Overlap of enriched intra-chromosomal interacting regions identified in biological replicate data generated using enzyme-based or sonication-based 4C-Seq approaches; C) Overlap of reproducible distal intra-chromosomal interacting regions from enzyme-based and sonication-based 4C-Seq approaches. 


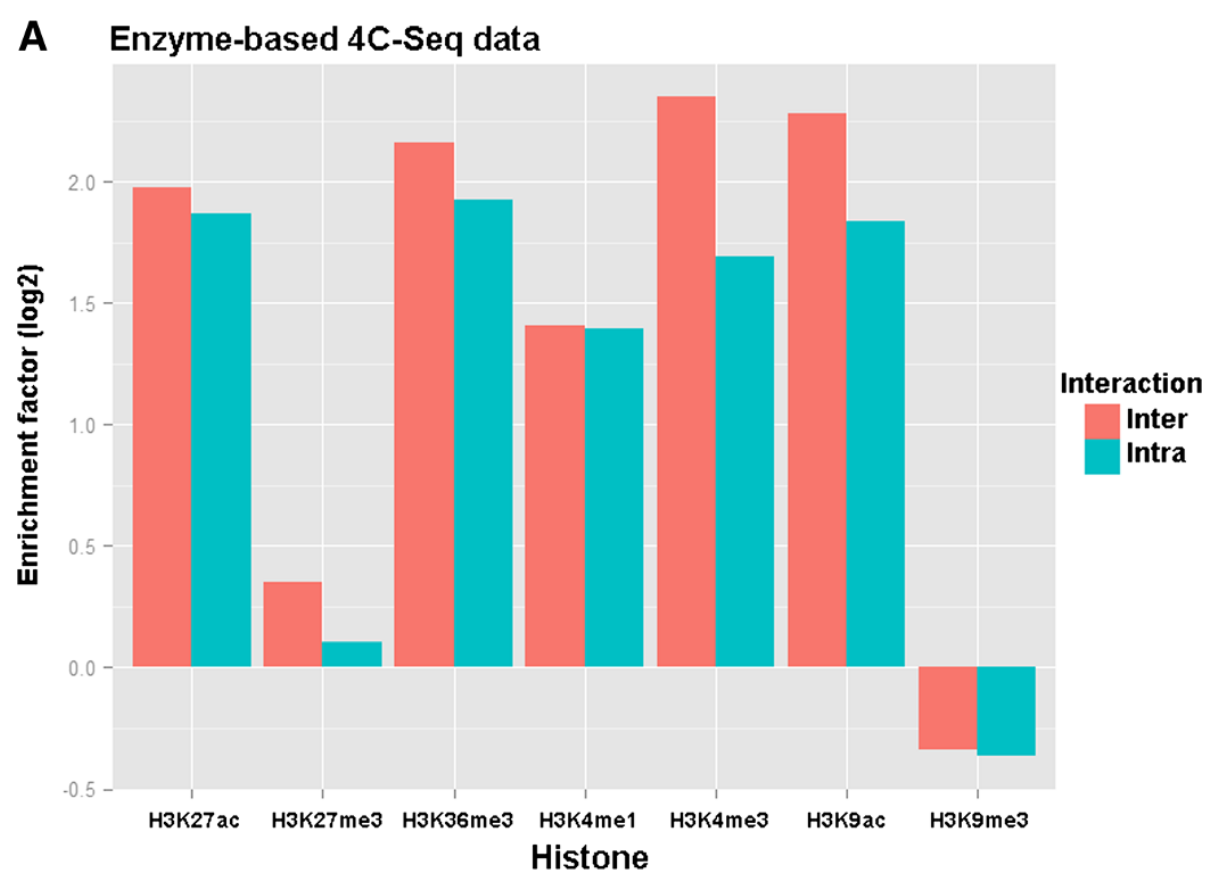

B Sonication-based 4C-Seq data

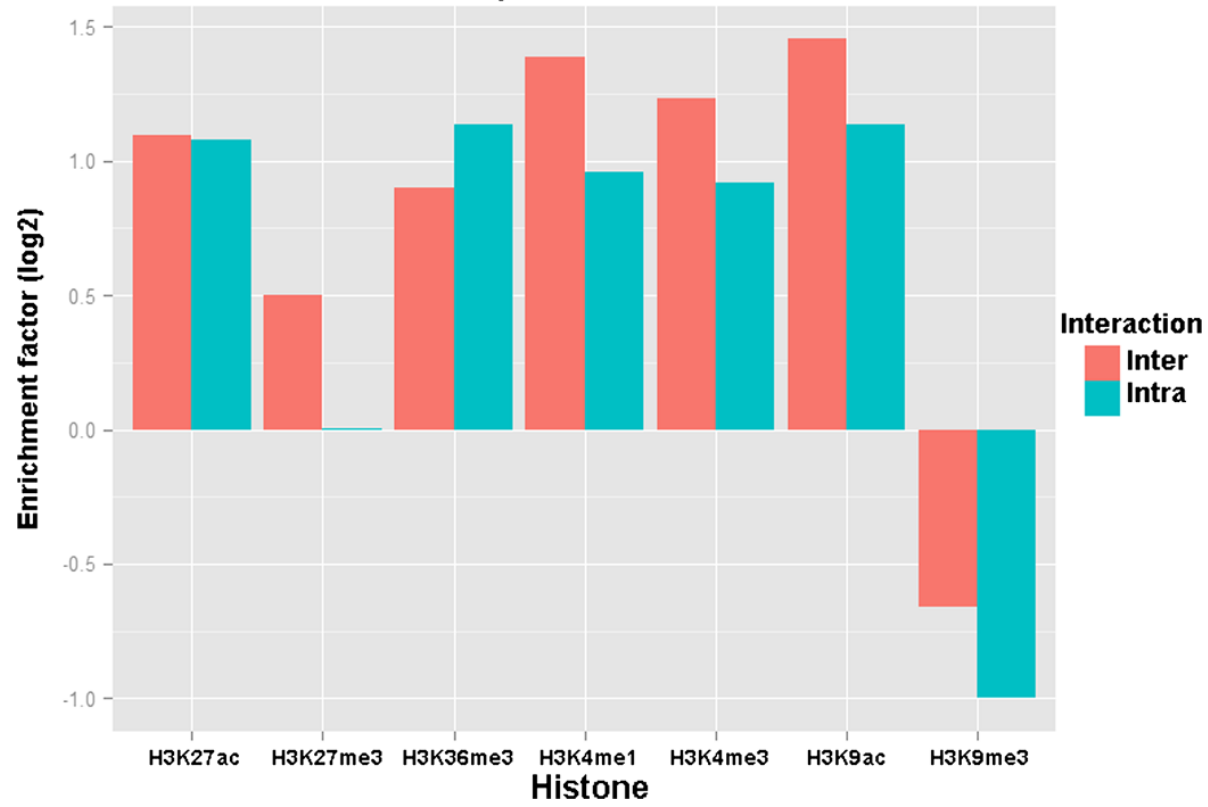

Figure 7 Enrichment analysis of histone marks. Bar plots of enrichment factor values of different histone marks around the interacting sites within the reproducible interacting regions. Enrichment factor was calculated as the observed sites in proximity to the ChIP-Seq peaks of a particular histone mark divided by the expected sites (random simulated across chromosome 17) close to that mark.

were calculated as background subtracted and plotted (Figure 8). Compared to randomly iterated genomic sites, the $4 \mathrm{C}$ interacting sites were moderately enriched with several transcription factors. Among them, transcription factors Oct4, Klf4, Esrrb, Tcfcp2i1 and Zfx showed statistically significant enrichment ( $\mathrm{p}<1 \times 10^{-10}$, unpaired WilcoxonMann-Whitney test), implying that these key pluripotency genes are mediating this enhancer interactome in mouse ES cells.

\section{Conclusions}

In summary, we presented $4 \mathrm{C}$-Seq data of an enhancer element in mouse ES cells, generated by two different methods: enzyme-based method and sonication-based 


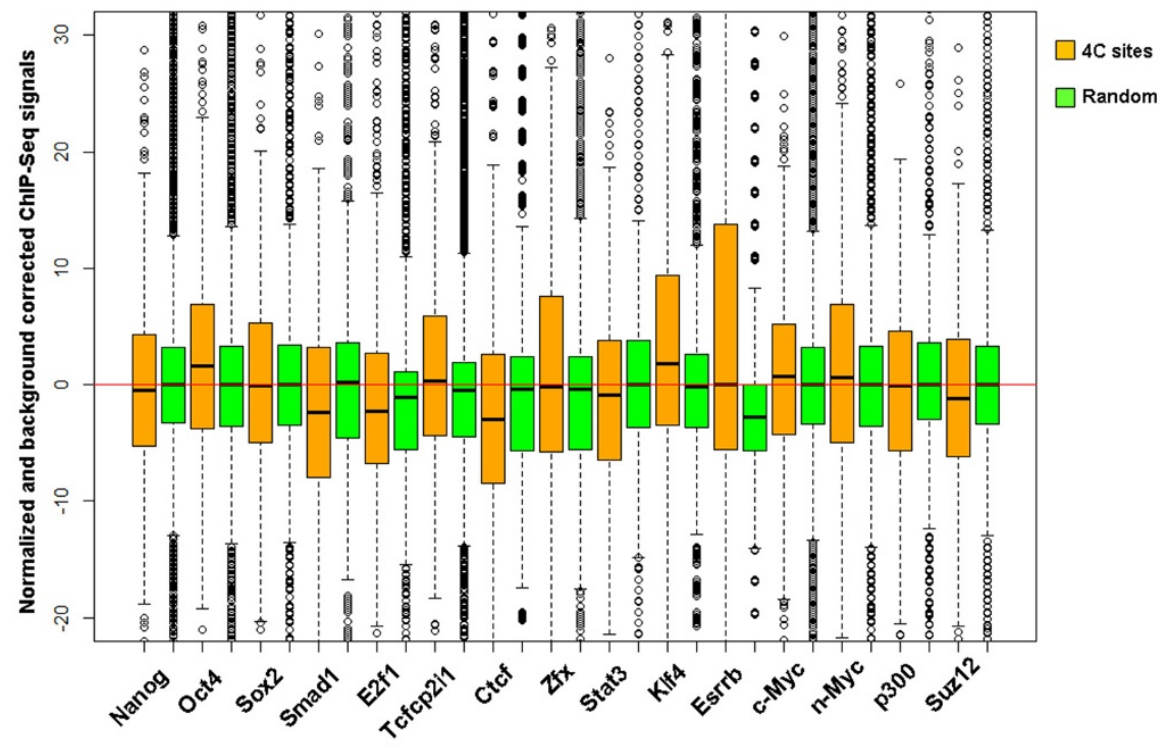

Figure 8 Enrichment analysis of DNA-binding proteins. Box plots showing distribution of normalized and background subtracted ChIP-Seq tag density of 15 DNA-binding proteins in mouse ES cells. Comparison was made between the 4C interacting sites (brown colored) and random iterated sites (green colored).

method. Our data showed consistency of the data generated by both methods for biological replicate samples. For inter-chromosomal interactions, both methods have similar reproducibility of enriched-interacting regions; however, for intra-chromosomal interactions, enzymebased method showed more frequent distal interactions and higher reproducibility. Both methods revealed that histone modifications related to gene activation were enriched in the interactomes. In addition, sonicationbased data uncovered several key pluripotency genes enriched around the interaction sites. Thus, we conclude that 1) Both enzyme-based and sonication-based $4 \mathrm{C}$-Seq technique are very useful tools for mapping long-range chromatin-chromatin interactions; and 2) 4C-Seq data together with ChIP-Seq data can help us elucidate molecular events surrounding a particular regulatory region in $3 \mathrm{D}$ space.

\section{Methods}

\section{Cell culture and $4 \mathrm{C}$ library preparation}

Mouse embryonic stem (ES) cell line E14 was grown in the culture dishes coated with $0.1 \%$ gelatin. A growth medium with Glasgow Minimum Essential Medium (GMEM) supplemented with $15 \%$ fetal bovine serum (FBS), $100 \mathrm{nM}$ nonessential amino acids, 1\% sodium pyruvate, $200 \mathrm{mM}$ glutamate, $1 \%$ penicillin streptomycin, $50 \mathrm{uM}$ b-mercaptoethanol and $10 \mathrm{ng} / \mathrm{mL}$ LIF was replaced every 24 hours to support ES cell growth. Immediately before $4 \mathrm{C}$ library preparation, 10 million cells were crosslinked with $1 \%$ fresh formaldehyde in the cell culture dishes, lifted and treated with Triton X100 buffer $(0.25 \%$ Triton X100, $10 \mathrm{mM}$ EDTA pH=8.0, $10 \mathrm{mM}$ Tris- $\mathrm{HCl}$ $\mathrm{pH}=8.0,100 \mathrm{mM} \mathrm{NaCl}, 1 \mathrm{x}$ protease inhibitor cocktail) to extract chromatins.

For sonication-based 4C library preparation, the isolated chromatin pellets were re-suspended in SDS lysis buffer

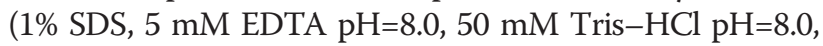
$1 \mathrm{x}$ protease inhibitor cocktail) and sonicated to an average size of $500 \mathrm{bp}$. The diluted chromatin fragments were blunt-end repaired and ligated with T4 ligase for 24 hours at $4^{\circ} \mathrm{C}$, followed by reverse crosslinking at $65^{\circ} \mathrm{C}$ for 20 hours with proteinase $\mathrm{K}$. The purified DNA served as PCR template for $4 \mathrm{C}$ library construction. After nested PCR amplification for library construction, the purified PCR products (majority with size ranging from $500 \mathrm{bp}$ to $1 \mathrm{~kb}$ ) were further sonicated to small DNA fragments with an average size of $200 \mathrm{bp}$ for sequencing. The Illumina HiSeq2000 Sequencer was used to perform paired-end sequencing with $90 \mathrm{bp}$ read length.

For enzyme-based 4C library preparation, two rounds of enzyme digestions were carried out using HindIII and DpnII restriction enzymes respectively. The experimental procedure strictly followed the published protocol [35]. The bar-coded DNA libraries were subject to single-end sequencing with 50 bp read length using the Illumina HiSeq2000. 


\section{Processing of $4 \mathrm{C}$-Seq data}

For sonication-based 4C-Seq data, Burrows-Wheeler Aligner (BWA) [29] with the "samse" option was employed for aligning paired-end data separately to the reference genome assembly $\mathrm{mm} 9$. Alignment files for both ends were further merged using SAMtools [31], followed by a PERL script to select only uniquely aligned read pairs (BWA mapping score $\geq 1$ ), where one end aligned to the bait region and the other end aligned to other regions. Finally, tags within a $100 \mathrm{bp}$ range were merged as a unique interacting site using BEDTools [36]. Singlet interacting sites were considered as background noise and removed.

For enzyme-based $4 \mathrm{C}$-Seq data, the sequencing reads with 5 ' end aligned to the forward inverse PCR primer sequence were selected. The rest part of the selected reads (including HindIII sites) was mapped to the $\mathrm{mm} 9$ assembly using BWA to locate ligation sites in the genome. The mapped ligated HindIII sites were further matched to a reduced genome with the locations of all HindIII sites included.

\section{Statistical model to identify enriched interacting regions for sonication-based 4C-Seq data}

A statistical model was built to identify interacting regions that exhibit a higher frequency of interactions with the bait than expected from random collision of the chromosomes [18]. For every interacting site $i$ on chromosome W (length $\mathrm{L}_{\mathrm{W}}$ ), the number of interacting sites within a certain window $w$ with length $l_{\mathrm{W}}( \pm 1 \mathrm{Mb}$ for inter-chromosomal interactions and $\pm 200 \mathrm{~kb}$ for intra-chromosomal interactions in our study) from the analyzed site was counted as $\mathrm{C}_{i, w}$, and a $\mathrm{z}$-score was calculated as an enrichment score:

$$
z_{i}=\frac{\left(C_{i W}-\mu_{W}\right)}{\sqrt{\mu_{W}\left(1-P_{W}\right)}}
$$

in which $\mathrm{p}_{\mathrm{W}}=\mu_{\mathrm{W}} / \mathrm{l}_{\mathrm{W}}$ ( $\mu_{\mathrm{W}}$ is the expected number of interacting sites in window $w$ on chromosome W).

Statistical significance was further assigned to each interacting site by a FDR-based approach. Briefly, we randomly permutated calculated z-score data for every chromosome 100 times, and chose interacting sites with a false discovery rate (FDR) $\leq 5 \%$ or FDR $\leq 20 \%$ as positively interacting sites for inter-chromosomal or intra-chromosomal interactions. FDR for each site was calculated by counting the number of randomly permutated Z-scores that are above experimentally determined Z-score. All the interacting sites within $\pm 1 \mathrm{Mb}$ range of a positively interacting site were clustered as an enriched interacting domain. Overlapping interacting domains were further merged together.

\section{ChIP-Seq data}

The ChIP-Seq peak data of histone variants for mouse ES cells were retrieved from the ENCODE database (http://genome.ucsc.edu/ENCODE). The ChIP-Seq raw read files of 15 DNA-binding proteins were downloaded from the GEO database (accession number GSE11431).

\section{Statistical analysis}

Statistical analysis was executed and plotted using the $\mathrm{R}$ software suite (http://www.r-project.org/). The conversion of genomic coordinates between different genome assemblies was done by liftover software tool (http://www.genome.ucsc.edu/).

\section{Availability of supporting data}

The sequencing data has been deposited to the GEO database (accession number GSE43776, GSE45418).

\section{Additional files}

Additional file 1: Summary of mapping results of 4C-Seq data.

Additional file 2: Figure S1. Distribution of read counts at all inter-chromosomal interaction sites. Data generated from the biological replicates BR1 and BR2 were compared. Both enzyme-based and sonication-based data were included in the plots.

Additional file 3: Figure S2. Reproducibility of inter-chromosomal interactions at different resolutions. Density scatter plots of inter-chromosomal interactions between the biological replicate data at resolutions of (A), $1 \mathrm{Mb}$ for sonication-based data; (B), 250 6-bp cutter sites for enzyme-based data; (C), 500 kb for sonication-based data; (D), 125 6-bp cutter sites for enzyme-based data.

\section{Competing interest}

All authors declare that they have no competing interest.

\section{Authors' contributions}

FG, WL and KW designed the project. FG performed the research and implemented the pipeline. ZW prepared the enzyme-based 4C library samples for sequencing. FG, WL and KW wrote the paper. All authors read and approved the final manuscripts.

\section{Acknowledgements}

The study is supported by departmental start-up funds from the Zilkha Neurogenetic Institute (K.W.), NIH grants R01 HG006465 (K.W.) and 5R01NS067213-02 (W. L.). We thank all members in the Lu lab and Wang lab for helpful comments and suggestions.

Received: 8 October 2012 Accepted: 20 May 2013

Published: 24 May 2013

\section{References}

1. Dekker J, Rippe K, Dekker M, Kleckner N: Capturing chromosome conformation. Science 2002, 295:1306-1311.

2. Lieberman-Aiden E, van Berkum NL, Williams L, Imakaev M, Ragoczy T, Telling A, Amit I, Lajoie BR, Sabo PJ, Dorschner MO, et al: Comprehensive mapping of long-range interactions reveals folding principles of the human genome. Science 2009, 326:289-293.

3. Dixon JR, Selvaraj S, Yue F, Kim A, Li Y, Shen Y, Hu M, Liu JS, Ren B: Topological domains in mammalian genomes identified by analysis of chromatin interactions. Nature 2012, 485:376-380.

4. Nora EP, Lajoie BR, Schulz EG, Giorgetti L, Okamoto I, Servant N, Piolot T, van Berkum NL, Meisig J, Sedat J, et al: Spatial partitioning of the regulatory landscape of the X-inactivation centre. Nature 2012, 485:381-385. 
5. Kalhor R, Tjong H, Jayathilaka N, Alber F, Chen L: Genome architectures revealed by tethered chromosome conformation capture and population-based modeling. Nat Biotechnol 2011, 30:90-98.

6. Carroll JS, Liu XS, Brodsky AS, Li W, Meyer CA, Szary AJ, Eeckhoute J, Shao W, Hestermann EV, Geistlinger TR, et al: Chromosome-wide mapping of estrogen receptor binding reveals long-range regulation requiring the forkhead protein FoxA1. Cell 2005, 122:33-43.

7. Kumar PP, Bischof O, Purbey PK, Notani D, Urlaub H, Dejean A, Galande S: Functional interaction between PML and SATB1 regulates chromatinloop architecture and transcription of the MHC class I locus. Nat Cell Biol 2007, 9:45-56

8. Pan YF, Wansa KD, Liu MH, Zhao B, Hong SZ, Tan PY, Lim KS, Bourque G, Liu $E T$, Cheung E: Regulation of estrogen receptor-mediated long range transcription via evolutionarily conserved distal response elements. J Biol Chem 2008, 283:32977-32988.

9. Horike S, Cai S, Miyano M, Cheng JF, Kohwi-Shigematsu T: Loss of silentchromatin looping and impaired imprinting of DLX5 in Rett syndrome. Nat Genet 2005, 37:31-40.

10. Cai S, Lee CC, Kohwi-Shigematsu T: SATB1 packages densely looped, transcriptionally active chromatin for coordinated expression of cytokine genes. Nat Genet 2006, 38:1278-1288

11. Fullwood MJ, Liu MH, Pan YF, Liu J, Xu H, Mohamed YB, Orlov YL, Velkov S, Ho A, Mei PH, et al: An oestrogen-receptor-alpha-bound human chromatin interactome. Nature 2009, 462:58-64.

12. Handoko L, Xu H, Li G, Ngan CY, Chew E, Schnapp M, Lee CW, Ye C, Ping $J$, Mulawadi F, et al: CTCF-mediated functional chromatin interactome in pluripotent cells. Nat Genet 2011, 43:630-638

13. Li G, Ruan X, Auerbach RK, Sandhu KS, Zheng M, Wang P, Poh HM, Goh Y, Lim J, Zhang J, et al: Extensive promoter-centered chromatin interactions provide a topological basis for transcription regulation. Cell 2012, 148:84-98.

14. Zhao Z, Tavoosidana G, Sjolinder M, Gondor A, Mariano P, Wang S, Kanduri C, Lezcano M, Sandhu KS, Singh U, et al: Circular chromosome conformation capture (4C) uncovers extensive networks of epigenetically regulated intra- and interchromosomal interactions. Nat Genet 2006, 38:1341-1347.

15. Ren $L$, Wang $Y$, Shi M, Wang $X$, Yang Z, Zhao Z: CTCF mediates the celltype specific spatial organization of the Kcnq5 locus and the local gene regulation. PLoS One 2012, 7:e31416.

16. Simonis M, Klous $P$, Splinter $E$, Moshkin $Y$, Willemsen $R$, de Wit $E$, van Steensel $B$, de Laat W: Nuclear organization of active and inactive chromatin domains uncovered by chromosome conformation captureon-chip (4C). Nat Genet 2006, 38:1348-1354.

17. Noordermeer D, Leleu M, Splinter E, Rougemont J, De Laat W, Duboule D: The dynamic architecture of Hox gene clusters. Science 2011, 334:222-225

18. Splinter E, de Wit E, Nora EP, Klous P, van de Werken HJ, Zhu Y, Kaaij L, van ljcken W, Gribnau J, Heard E, de Laat W: The inactive X chromosome adopts a unique three-dimensional conformation that is dependent on Xist RNA. Genes Dev 2011, 25:1371-1383.

19. Robyr D, Friedli M, Gehrig C, Arcangeli M, Marin M, Guipponi M, Farinelli L, Barde I, Verp S, Trono D, Antonarakis SE: Chromosome conformation capture uncovers potential genome-wide interactions between human conserved non-coding sequences. PLoS One 2011, 6:e17634.

20. Gondor A, Rougier C, Ohlsson R: High-resolution circular chromosome conformation capture assay. Nat Protoc 2008, 3:303-313.

21. Simonis M, Kooren J, de Laat W: An evaluation of 3C-based methods to capture DNA interactions. Nat Methods 2007, 4:895-901.

22. Fullwood MJ, Ruan Y: ChIP-based methods for the identification of longrange chromatin interactions. J Cell Biochem 2009, 107:30-39.

23. van de Werken HJ, Landan G, Holwerda SJ, Hoichman M, Klous P, Chachik R, Splinter E, Valdes-Quezada C, Oz Y, Bouwman BA, et al: Robust 4C-seq data analysis to screen for regulatory DNA interactions. Nat Methods 2012, 9:969-972.

24. Auerbach RK, Euskirchen G, Rozowsky J, Lamarre-Vincent N, Moqtaderi Z, Lefrancois P, Struhl K, Gerstein M, Snyder M: Mapping accessible chromatin regions using Sono-Seq. Proc Natl Acad Sci U S A 2009, 106:14926-14931.

25. Teytelman L, Ozaydin B, Zill O, Lefrancois P, Snyder M, Rine J, Eisen MB: Impact of chromatin structures on DNA processing for genomic analyses. PLoS One 2009, 4:e6700.

26. Huang PY, Han Y, Handoko L, Velkov S, Wong E, Cheung E, Ruan X, Wei C, Fullwood MJ, Ruan Y: Sonication-based Circular Chromosome
Conformation Capture with next-generation sequencing analysis for the detection of chromatin interactions. Protocol Exchange: Protocol 2010.

27. Takahashi K, Yamanaka S: Induction of pluripotent stem cells from mouse embryonic and adult fibroblast cultures by defined factors. Cell 2006, 126:663-676.

28. Sexton T, Yaffe E, Kenigsberg E, Bantignies F, Leblanc B, Hoichman M, Parrinello $H$, Tanay A, Cavalli G: Three-dimensional folding and functional organization principles of the Drosophila genome. Cell 2012, 148:458-472.

29. Li H, Durbin R: Fast and accurate short read alignment with BurrowsWheeler transform. Bioinformatics 2009, 25:1754-1760.

30. Li G, Fullwood MJ, Xu H, Mulawadi FH, Velkov S, Vega V, Ariyaratne PN, Mohamed YB, Ooi HS, Tennakoon C, et al: ChIA-PET tool for comprehensive chromatin interaction analysis with paired-end tag sequencing. Genome Biol 2010, 11:R22.

31. Li H, Handsaker B, Wysoker A, Fennell T, Ruan J, Homer N, Marth G, Abecasis $G$, Durbin R: The sequence alignment/map format and SAMtools. Bioinformatics 2009, 25:2078-2079.

32. Lanctot C, Cheutin T, Cremer M, Cavalli G, Cremer T: Dynamic genome architecture in the nuclear space: regulation of gene expression in three dimensions. Nat Rev Genet 2007, 8:104-115.

33. Schoenfelder S, Sexton T, Chakalova L, Cope NF, Horton A, Andrews S, Kurukuti S, Mitchell JA, Umlauf D, Dimitrova DS, et al: Preferential associations between co-regulated genes reveal a transcriptional interactome in erythroid cells. Nat Genet 2010, 42:53-61.

34. Chen X, Xu H, Yuan P, Fang F, Huss M, Vega VB, Wong E, Orlov YL, Zhang W, Jiang J, et al: Integration of external signaling pathways with the core transcriptional network in embryonic stem cells. Cell 2008, 133:1106-1117.

35. Splinter E, de Wit E, van de Werken HJ, Klous P, de Laat W: Determining long-range chromatin interactions for selected genomic sites using 4C-seq technology: From fixation to computation. Methods 2012, 58:221-230.

36. Quinlan AR, Hall IM: BEDTools: a flexible suite of utilities for comparing genomic features. Bioinformatics 2010, 26:841-842.

doi:10.1186/1471-2164-14-345

Cite this article as: Gao et al:: Comparative analysis of 4C-Seq data generated from enzyme-based and sonication-based methods. BMC Genomics 2013 14:345.

\section{Submit your next manuscript to BioMed Central and take full advantage of:}

- Convenient online submission

- Thorough peer review

- No space constraints or color figure charges

- Immediate publication on acceptance

- Inclusion in PubMed, CAS, Scopus and Google Scholar

- Research which is freely available for redistribution 